THE DIGTTAL DATA ACQUISITION CHAIN AND THE COSMIC RAY TRIGGER SYSTEM FOR THE SLD WARM IRON CALORMMETER'†

\author{
A. Benvenuti \\ INFN Sezione di Bologna, I-40126 Bologna, Italy
}

L. Piemontese

INFN Sezione di Ferrara \& Univensita di Ferrara, 1-44100 Ferrara, Ihaly

A. Calcaterra, R. De Sangro, P. De Simone

Leboratori Nazionali di Frascati dell 'NFN, I-00044 Frascati (Roma), ttaly

P.N. Burrows. S.L. Curtwright, S. Gonzales, A. Lath, U. Schneckloth, D.C. Williams, J.M. Yanartino

Massachusetts Institute of Technology. Cambridge, MA 02139 USA

N. Becchetta, D. Bisello, A. Castro, S. Galvagni, M. Loreti, L. Pencara, J. Wyss INFN Sezione di Padova \& Universitż di Padava, I-35100 Padova, flafy

B. Alpat, G.M. Bilei, B. Checcucci, R. Dell'Orso, M. Pauluzyi, L. Servoli INFN Sezione di Perugia \& Universits di Perugia, I-06100 Perugia, Italy

M. Carpinelli, R. Castaldi, U. Cazzola, C. Vannini, P.G. Verdini

INFN Sezione di Pisa \& Universith di Pisa, I-56010 San Piero a Grado (Pisa), Ilaly

R.L. Messner, R.W. Zdarko

Stanford Linear Accelerator Ceater, Stanford Univetsity, Stanford, CA 94309 USA

\title{
J.R. Johnson
}

University of Wisconsin, Madiron, WI 53706 USA

In wited toik presented at the the Piva Meeting on Advanced Detectors:

Frontier Detectors for Frontier Physics,

Lo Biodola, Isola d'Elba, IHaly, May 21-26, 1989

\footnotetext{
Work aupporled in part by Department of Enetgy (USA) contracts DE-AC02-76ER03069 (MIT). DE-AC03-76SF00515 (SLAC), and DE-AC02-76ER008B1 (UW), and by the Litituto Nasionale d: Finies Hueleare (Italy).

tTall presented by Piero G. Veedigi.
} 


\section{Abstract}

The entire data-arquiuizion chain, from the custom-made front-end electronics to the Fustbus readout and data-reduction module, for the digital readout of the SLD [1] limited atreamer tube Warm Iron Calorimeter and Muon Identifier is deacribed. Also described is a Fastbuy Cosmic Logic Unit being developed to achieve the capability of reading cosmic ray events, also during the inter-crossing time, for opparatus monitoring and calibration purposes.

\section{Introduction}

The SLD Warm Iron Calorimeter and Muon Identifier (WIC) [2] is a structure of $5 \mathrm{~cm}$ thick iron plates interleaved with standard $(0.9 \mathrm{~mm}$ cell vize) plastic limited streamer tubes. To fully exploit the muon identification and tracking potential of the device, a great number $\left(\approx 10^{\circ}\right)$ of teackiag channeh ("atripa," see Fig. 1) must be read; additionally, to echicve maximum hermeticity, the number of outgoing dots tranafer links must be minimized; lost, it is desirable to apply zero-s'jppression and possibly clustering and miomapping correction algorithms to the data $4000 \mathrm{p}$ as fesulble along the readout chain. This obviously poses heavy restrictions on the cost and the encumbrance of the front-end digital electronics, on the resdout mode and on the data reduction and preanalysis capabilities of the data acquisition module.

The custom desigr D779 CMOS chip-a joint project of SGS [3] and of the lotituto Nazionale per bs Fisica Nucleare (INFN) - contains four common threshold diacriminators, each followed by a one-shot, plus a 4-bit wide shift regiater (Fig, 2) having daisy chaining capabilities in 16.pin dual-in-line micro-die package. The chip has been adopled as a building block for the frontend electronica. The capability of the D779 of givits a Digor-the fal wired-Or combination of the discriminator outputs-easily extended to form triggering information over aumbers of D779's, and has also been exploited to conetruet a comic ray trigger for the apporatus.

A dual-D779 hybrid with a fast two-stage, 50 times gain preanplifier $[4,5]$ has been developed joinlly with SGS to enable full detection efficiency operation evea if the chambers are at an operating point kubstantially lower than the limited streaner point.

The natural date etructure in such a shift-regioter-based environment is the daisy chain of all the D779's connected to the stripa of one plane of the apparatus. To minimize the number of outgoing dats (and incoming control) connections, a concentrator board ("splitler bourd") has also been developed [7] to link dsisy chaino belonging to the same cub-sub-aystem and having remole system configuration and diagnostic capabilities. The Splitter Bourd fentures a separate DAC threshold eetting for the discriminators of each of the ten planes served, independent ADC readout of the threshold waluch, and combinatory and majority logje whtch allows a sub-aub-oystem trigger to form which is then used by the Cosmic Logic Unit (CLU).

The Warm Iron Calorimeter Digital Readout Module (WICDRM) $[5,6]$ was also designed koeping well in mind the daisy chain structure characteristic of the front-end

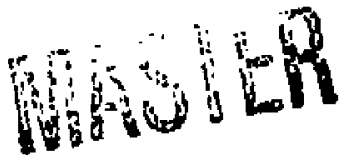


boards, which minimizes the number of connections with the interior of the detector while retaining the capability to perform two complete readout cycles per Stanford Linear Collider (SLC) pulse, even at the design maximum operating frequency of $180 \mathrm{~Hz}$. A MC68020 microprocessor [8] is used to perform a first stage of data reduction and apparatus monitoring exploiting the intercrossing readout. This choice for the $\mathrm{CPU}$ was dirtated by its powerful processing and input-output capabilities and full 32-bit architecture; which nicely matches the 32 -fold modularity of the front-end eleetronics and the availability of good software support.

\section{The Dront-End Flectronics}

The S1,D Advisory Committee requested that a solution be tuund for the WIC frontend electronics capable of operating with strip signals much smaller than those characteristic of the limited streamer regime, should detector deterioration advise in favor of a drastic reduction of the electrical field in the chambers. A two-stage preamplifier for the D779, using two very fast $2 \mathrm{N918}$ transistors for a preamplification of approximately 50 times but having the additional requirement of $a+12 \mathrm{~V}$ power supply, was tested and eventually selected.

Figure 3 summarizes part of the studies made on the threshold curve dependence upon the operating point of the limited streamer tubes in a cosmic ray setup. The lower scale shows the $V_{\text {th }}$ applied to the D779's, while the upper scale refers to the threshold voltage renormalized by the preamplification, showing therefore the equivalent pulse height of the strip signals. The complementary plot, showing the high voltage plateau at two different $V_{\text {th }}$ settings, is shown in Fig- 4, demonstrating how full efficiency can be attained operating the chambers over a large high voltage range, even in the proportional regime.

The digital front-end clectronics of the WIC calorimeter in the SLD detector comprises approximately 90,000 strip readout channels. A block diagram of the readout system is shown in Fig. 5. Eight channels of preamplifiery, discriminators, one-shots and shift-registers are packaged in a single hybrid; four hybrids are packaged in 32-channel boards, and all the boards of each layer of the detector (typically 10 boards/layer) are daisy-chained logether. Sulssequently groups of approximately 3000 rlinnels are further multiplexed in the splitter boards, and up to eight splitter boards are read by each WICDRM.

Mass production and testing of the boards (one of which is portrayod in Fig. 6) is now completed, and the front-end electronics is being installed on the dotector.

\section{The Splitter Board}

The front-end boards of the cham'er planes belonging to the same sub-sub-system, such as balf an octant of the barrel ( coffin") or a section of the endcaps, are grouped and powered, controlled and read by a splitter board. Each splitter board can handle up to ten planes. A block diagram is shown in Fig. 7. Through the daisy chains the Splitter Boards provide the front-end boards with regulated power and threshold voltages; upon 
the occurrence of a trigger, they provide the load signal, latehing the one-ghot outputs onto the shift register chain. During the readout they distribute the shift regigter clock and a test pattern. generated by the Fastbus seadout module which transfors to the? module the appropriate shift tegister data from the front-end boards.

To allow for the maximum flexibility in delector operation, each front-end daisy chain has an individual discrimninator threshold set by a l2-bit DAC. The DAC outputs in the $040 \mathrm{~V}$ range undergo a 100 times reduction in a passive network on the boards themselves to improve the signal-to-noise ratio while still transmitting the $V_{\text {th }}$ along the daisy chains, logether with TTL signals. If one also takes into account the 50 times amplification on the board, one then has an effective $V_{\text {th }}$ range of $100 \mu \mathrm{V}$ to $8 \mathrm{mV}$, while typical values used are in the neighborhood of $2 \mathrm{mV}$.

The module also contains combinatory and mejority logic circuits capable of forming a trigger signal from the Digor outputs of the individual planes serviced by the Splitter 13oard. The trigger logic has provisions for excluding and for requiring hite on individual plines, and for setling the ininimum number of planes which must be hit for a trigger to be formed. In order to ninimize the noise possibly induced by the Digor along the daisy chains, the voltage swing is kept at $500 \mathrm{mV}$ and converted on the splitter board to TTI. levels. The total driay between the arrival of a valid Digor combination and the output of a trigger signal is less than 50 nsec.

The timing for the splitter board operations is provided by a Timing and Control Module (TCM) [9], which in synchronized with the 119 MHz LINAC main frequency. Communicalions with the TCM use two identical sets of four lines, duplicated for reliability. A fiber optic translator board converts the fo. signals from and to the TCM 10 differential T'TL for up 10 eight splitter boards, earh of which responds to a unique selection address and can therefore receive separate configuration informalion and operating commands. The splitter boards belonging to the same subgroup receive the shift register master clock and the shift register test pattern from the WICDIRM througli one fiber optic link whose output, converted to differential TTL, is then distributed. Bach splitter board has two individual fiber optic links, one witb the WICDRM, carrying the shift register data from the front-end boards, and one to the Cosmic Logic Unit carrying the splitter board trigger signal. A prototype splitter board is currently functioning on the detector, and production of the 42 needed for the WIC is expected to bo: complete by early autumn this yoar.

\section{The Digital Reodout Module}

The design of the Fastbus readout module for the strip information-the WICDRMwas heavily influenced by the following requirements: mininization of the number of modules and connections needed for the whole detector; ability to perform the strip readout at twice the bearn crossing rate, not only scarching for $e^{+} e^{-}$annihilation data at the crossing itsplf but also examining cosmic ray events of even random noise in the inter-crossing time to monitor the front-cid functionality; and last but not least, maximization of the anount of data processing done within the module itaclf, thus reducing the laad on the other links in the data scquisition chain and the Fastbus traffic. 
This brought to the construrtion of an "intelligent," albeit slave, Fastbus modult, based on the powserfu] Motorola MC68020 microprocessor.

After the front-end one-stot information bas been latched upon reception of a lond signal into the daisy-chained shift-registers, the readout module proceeds to clock their contents, one long word at a time and at a $4 \mathrm{Mbit} / \mathrm{sec}$ rate, into eight parallel inpul bufters (see the bluck diagram of Fig. B) under the full control of the mirroprocressor. Once the buffers have bewn filled, a flag signals the proressor to latch the datn and immediately start a new 32-bit cycle. While waiting for the completion of the shifling operation, the processor can either transfer to RAM the full extent of the date rearl or start the preliminary claboration with a zero-suppression which may also include correcting for misnsapped cabling of the front-end bosdb, clustering, data formating and strip occupation histogramming. Furthermore, extensis. testing of the front-end electronics is also possible, and the integrity of the daisy chains can be verifed by injerting a preselected pattern at the far end and comparing it with the results of a readout cycle. Conmunication with the splicter boards goes through an $\mathrm{J} / \mathrm{O}$ card mounted on the Fastbus auxiliary backplane, which can be customized to convert the particular tranomission protocol chosen (optical links in the SLD experiment) to the TTL levels used within the module, thus allowing testing of the chains without reguiring the installation of all the components of the system. The production and testing of the six WICDRM modules noeded for the whole WIC is well under way and the first batch of two should substitute the prototype (see Fig. 9) which is curtently operating on the detector by utmmer 1989.

\section{The Cosmic Logie Unit}

To fully benefit from the dual-readout capability of the WIC atrip electronics by collecting cosmic ray events in parallel with the physies data taking, a CLU was desizned to collate the splitter boord trigger signals and form a versatile cosmic ray trigger. One of the goals was to be able to change the trigger configuration by simply downloading new trigger tables to the unit, possibly under the control of a Fastbus master module when the SLD detector is running in cosmic ray mode and the cosmic ray events undergo the fullest elaboration. Furthermore, logic was added to provide triggering information. in the form of inputs to a Fastbus latch, for the SID Trigger Supervisor unit when running in physics mode.

The inputs to the CLU are the triggering signals from the 42 splitter boards; since in sone cases the logical units forning the detector have been split among more than one splitter board, a first level of signal recombination is done in hardwart on the fiter uptic to TTL conversion board. A private bus carries the 32 signals thus obtained to ther CLU proper. At this point, the barrel accounts for 16 coffin signals, two colfine radially juxlaposed forming all octant, while each endcap is subdivided into six sections, and four signals correspond to the 45-degree chambers. A set of PALs is used to build the prestriggers, combining the coffins into octants, adding the two middle-plane signnls of earh endcap, summing the 45 -uegree signals for each side. and providing a batrel-top and a barrel-bottom sum of the three uppermost (lowermost) octants.

A first level of lookup tables, using two sets of $4 K \times 125-n s e c$ access tine HAMts, is 
addresned by subgroups of 12 of the pre-triggers and outputs subgroups of fcur Level 1 triggern. Three of the four RAMs reserved tor the cosmic ray trigger are currently used: the first and the second build "tight" and "loose" single and multiple barrel muon triggres: the third rombines cudcap and barrel-top. barrel bottom information into similar outputs. At the current stage little thought has bret given to the physies trigger .utput:s, but possibilities studied are a lack-to-back muon signal, single penetrating track signals, and lack-to-back tracks which only hit the innet coffins but do not exil the devector. One of the rearons for building as nuch flexibility as possible into tlir (IL.' is, of conrse, to ease the adaptation to the unknown envitonment of the SLD at the SLC.

\section{Conclusions}

The full data acquisition chain for the Muon Tracking System of the SLD is eturrently being installed and debugged with cosmic ray data. All the components respond well to the expectintions, and the threshold plateaus obtained in a test environment (Fig. 4) have been reproduced systematically at the detector octant scale, using the whole readout chain from the front-end boards to the dati-acquisition host, thereby demonatrating the functionality of thie systen. By autumn 1989, the whole WIC detector should he completely instrumented for the strip readout and operational as a muon identifier and tracker, ready for stand-alone cosmic ray data taking.

\section{DISCLAIMER}

This report was prepared as an account of work sponsored by an agency of the United States Government. Neither the United States Government nor any agency thereof, nor any of their employees, makes any warranty, express or implied, or assumes any legal liability or responsibility for the accuracy, compleleness, or usefulness of any informatior., apparatus, product, or process disclosed, or represents that its use would not infringe privately owned rights. Refer. ence herein to any specific commercial product, process, or service by trade name. trademark, manufacturer, or otherwise does not necessarily constitute or imply its endorsement, recommendation, or favoring by the United States Government or any agency thereol. The views and opinions of authors expressed herein do not necessarily state or reflext those of the United Slates Government or any agency thereor. 


\section{References}

[1] SL.D Design Report, SLAC Report 273, (1984).

[2] A.C. Brnvenuti et al., Nucl. Instr. and Metl. A276, 94 (1089).

[3] SOS-Microelettronica S.p.A. - Milano, Italy.

[4] F. Heroncini et al., SLD-New Dehetor Note 154 (1986).

F. Broncini et al., INFN/TC-86/17 (1980).

F. Beroneini el al., IEEE Trans. Nucl. Sci. NS-35, л.l, 311 (1988).

[5] F, Beroncini et al, Nucl. Instr. and Meth. A277, 222 (1989).

[6] G.M. Bilei $e t$ al., IEEE Trans. Nucl. Sci. NS-35, n.1, 282 (1988).

[7] N. Bacchetta ef al., submitted to IEEE Trans. Nucl. Sci. (1989).

[8] MC68020 32-bit Micoprocessor User's Manual, Second Edition, (Prentice-11all. New Yor'., 1965).

19] S. MacKenzie, "SLD LAC Timing Unit," SLD Intcrnal Note. 


\section{Figures}

l'ig. J. Sl.d) WIC sirramer tubes.

Fig. $2 . \quad$ lnternal strueture of the D779.

Fig. 3. 'Thresloold voltage plateau.

Fig. 4. High voltage plateau.

Fig. 5. The SID-WIC strip data chain.

17ig. 6. Pholograph of a 32-rhannel front-and board.

Fig. 7. Block diagram of the splitter board.

Fig. 8. The WICDlla fromt-end processor.

Fig. 9. Photograph of the WICDRM prototype. 


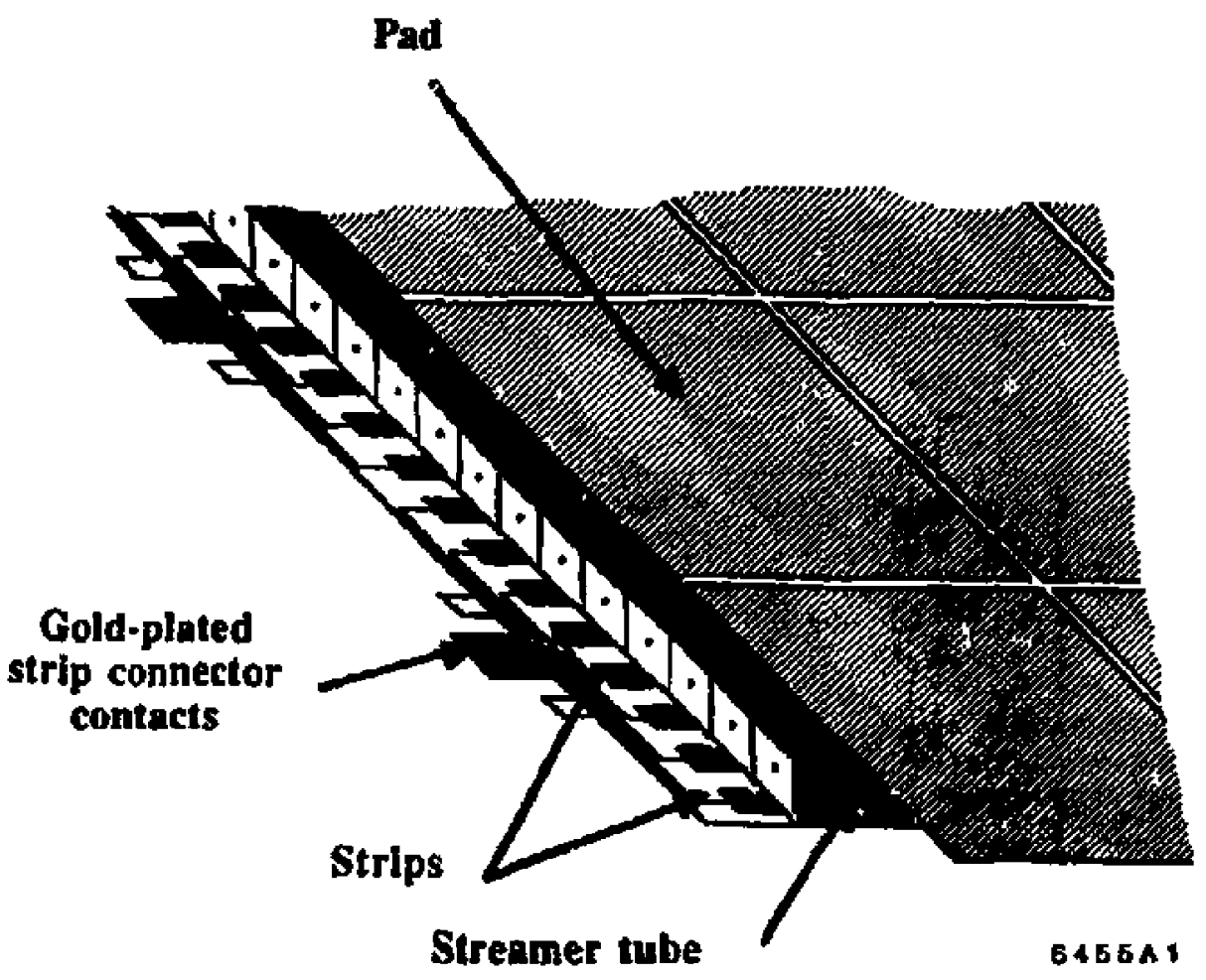

Fig. 1 


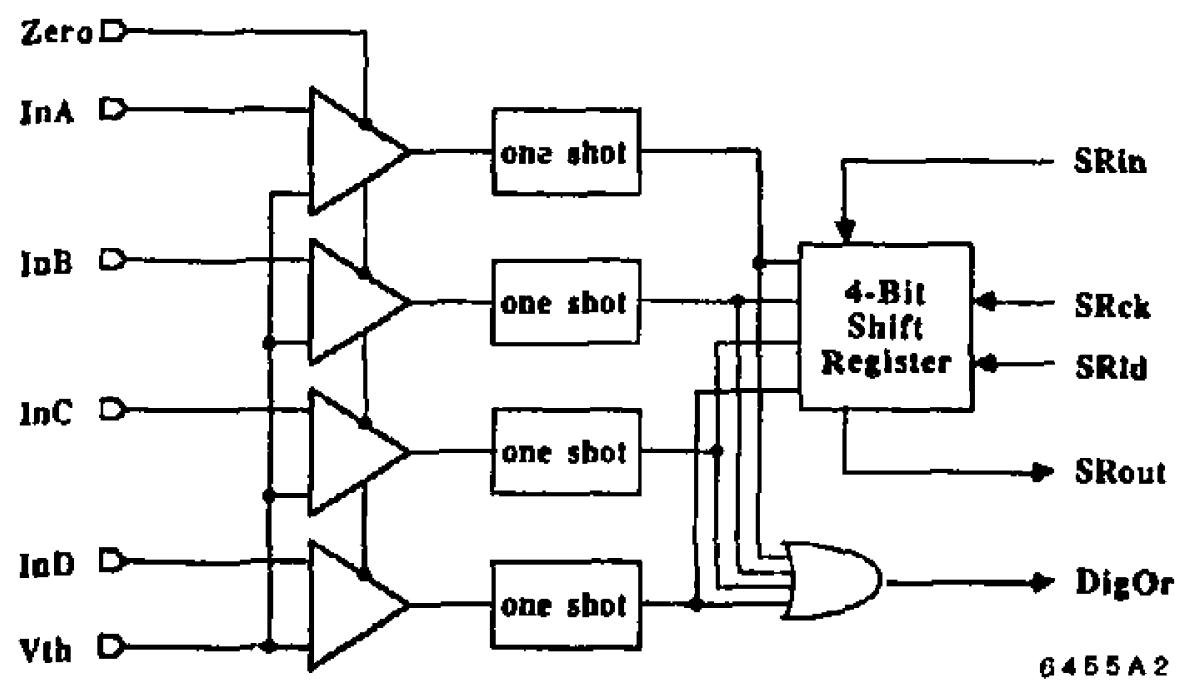

Fig. 2 


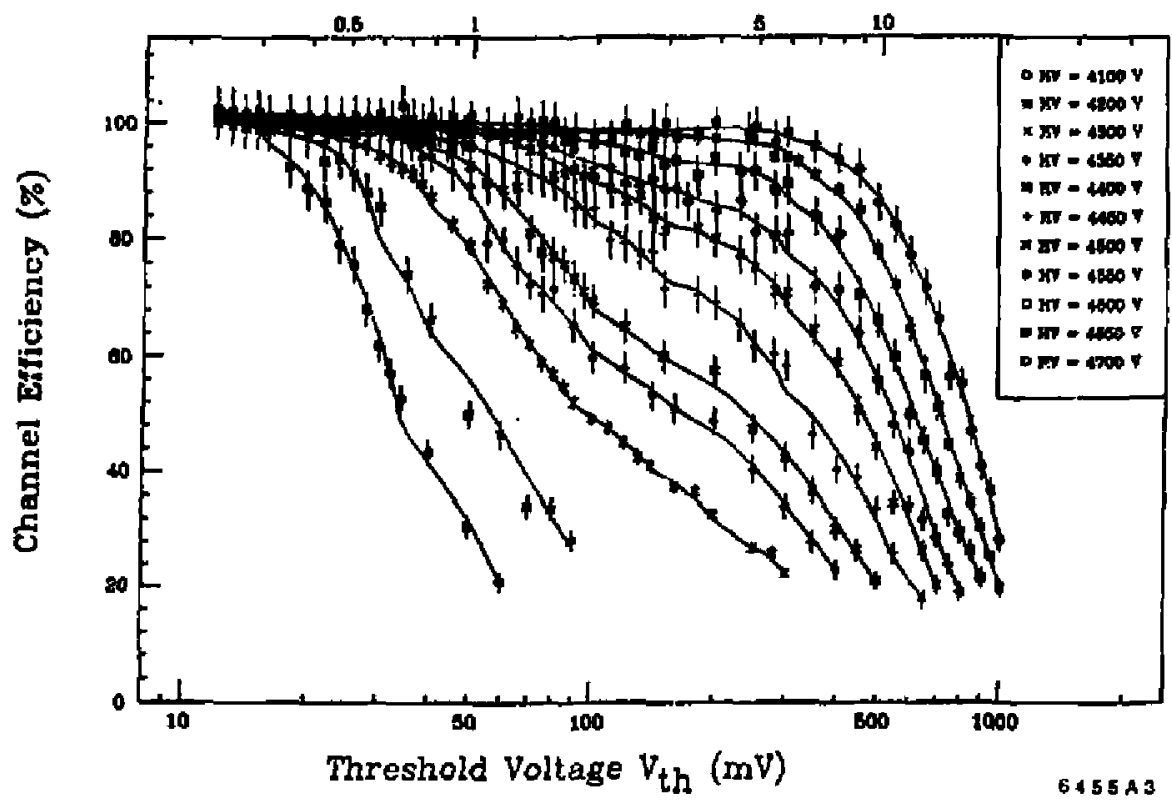

Fig 3 


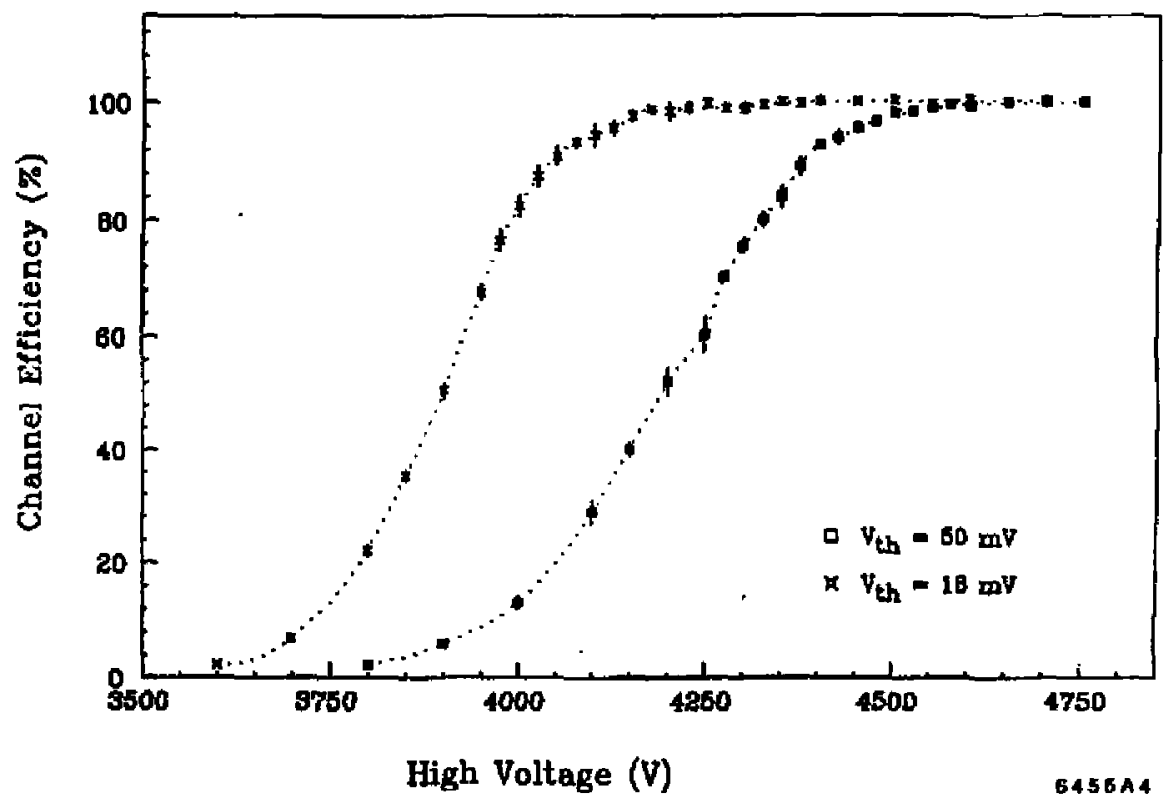

Fig. 4 


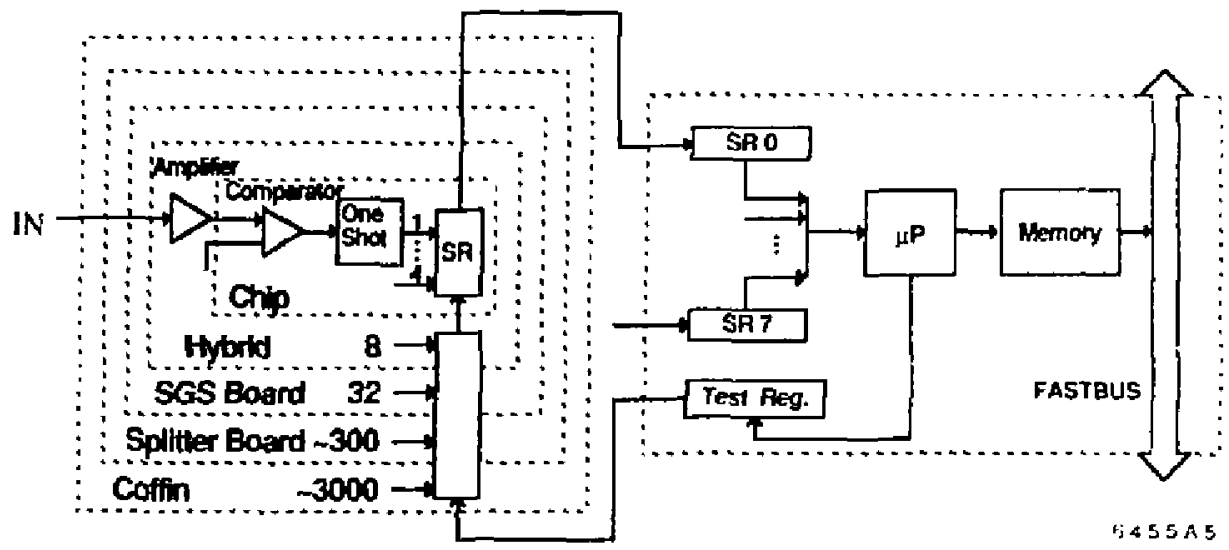

Fig. 5 


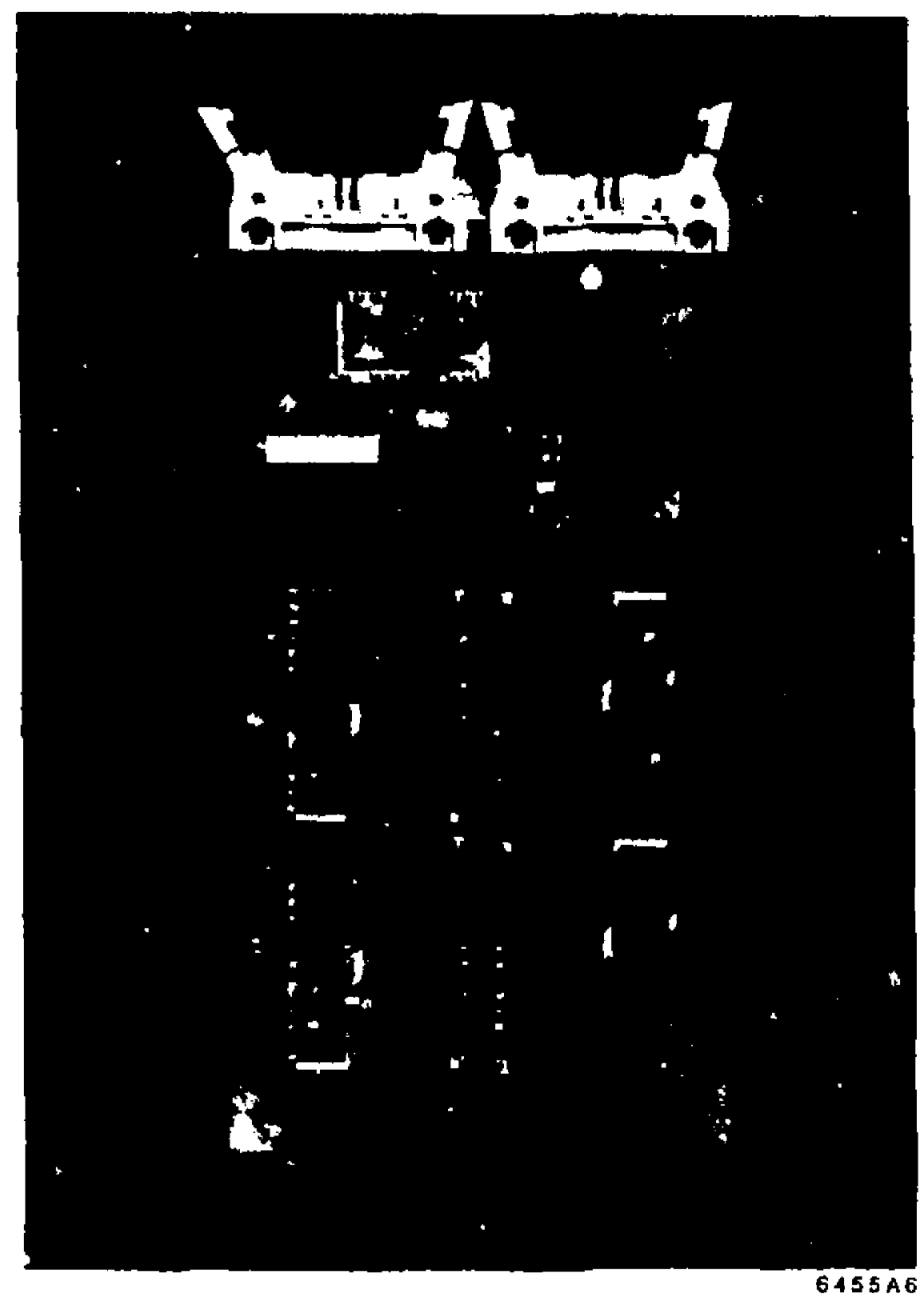

Fig. 6 


\section{Splitter Board block diagram}

To Ththinedns

To DNST-CHNHA

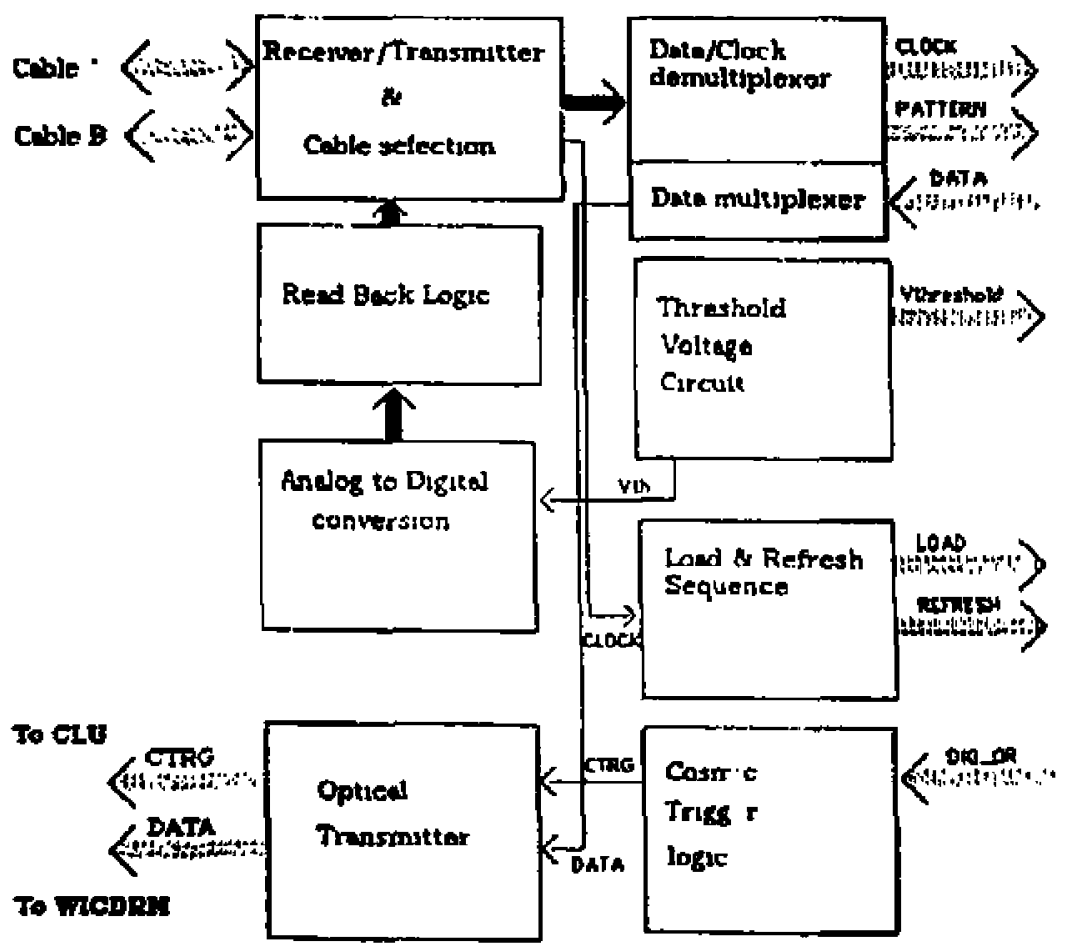

Fig. 7 


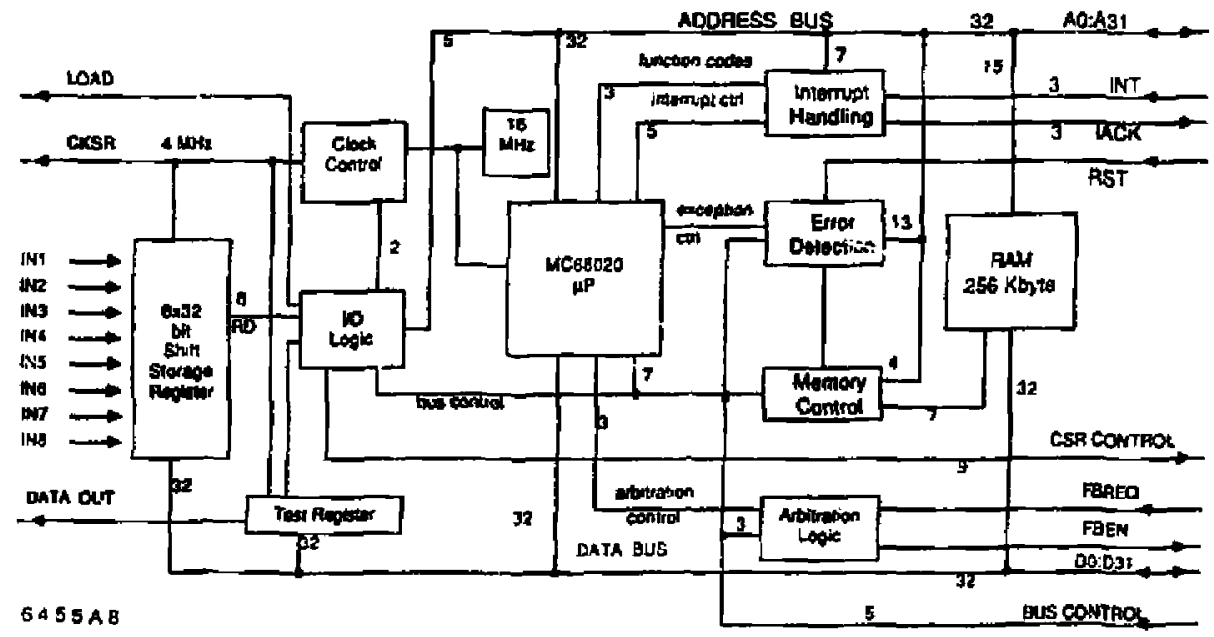

Fig. 8 
